\title{
Estandarización en la Observación y Clasificación de Lesiones Epiteliales Premalignas y Malignas
}

\author{
Standardization in the Observation and Classification \\ of Premalignant and Malignant Epithelial Lesions
}

*Juan Carlos Cuevas González; *Ixchel Maya García; *Francisco Germán Villanueva Sánchez;

${ }^{* * *}$ Luis Alberto Gaitán Cepeda \& ${ }^{* * *}$ Elba Rosa Leyva Huerta

CUEVAS G. J. C.; MAYA G. I.; VILLANUEVA S. F. G.; GAITÁN, C. L. A. \& LEYVA, H. E. R. Estandarización en la observación y clasificación de lesiones epiteliales premalignas y malignas. Int. J. Morphol., 29(3):706-710, 2011.

RESUMEN: Los criterios histológicos para determinar el grado de displasia, la clasificación de Broders y el frente de invasión tumoral (FIT) son parámetros subjetivos no cuantificables que pueden indicar el grado de evolución de displasias y carcinomas. Un factor importante a considerar durante la valoración histológica, es la variabilidad del diagnóstico entre patólogos. El objetivo es estandarizar los criterios y determinar la variabilidad intra e inter observador en el diagnóstico de displasias y COCE. Se seleccionaron y estandarizaron los criterios morfológicos para el diagnóstico y se revisaron los casos seleccionados aleatoriamente por tres patólogos bucales (30 displasias y 30 carcinomas) del Laboratorio de Patología Clínica y Experimental de la DEPeI de la FO, UNAM. Cada patólogo analizó y registró los parámetros establecidos para displasia, COCE y FIT en 2 ocasiones. Se aplicó el test Kappa para valorar la concordancia intra e inter observador. El Observador $1 \mathrm{v} / \mathrm{s}$ el 2 obtuvo una concordancia para COCE de 0,75 y en displasias de 0,60 e intraobservador de 0,90. El observador 2 v/s el 3 presentó una concordancia para COCE de 0,75 y en displasias de 0,59 e intraobservador de 0,91 . El Observador 3 Vs el 1 tuvo una concordancia para COCE de 0,77 , y en displasias de 0,59 e intraobservador de 0,92. La concordancia intraobservador e interobservador en COCE fue de buena a excelente, pero en displasias fue aceptable confirmando que su evaluación presenta mayor grado de dificultad. Con una adecuada estandarización se puede obtener una buena concordancia entre patólogos.

PALABRAS CLAVE: Estandarización de criterios; Variabilidad interobservador; Variabilidad Diagnóstica; Lesiones epiteliales.

\section{INTRODUCCIÓN}

Las neoplasias malignas de origen epitelial constituyen el $90 \%$ de todos los cánceres de cabeza y cuello. El diagnóstico preciso así como su graditaje son fundamentales para un correcto tratamiento que influye en la sobrevida de los pacientes Los criterios histológicos para determinar el grado de displasia, la clasificación de Broders y el frente de invasión tumoral (FIT) son parámetros subjetivos no cuantificables que pueden indicar el grado de evolución de displasias y carcinomas. A pesar de las múltiples técnicas utilizadas para establecer un diagnóstico, la observación microscópica por parte del patólogo sigue siendo la herramienta de mayor utilidad en el diagnóstico de lesiones premalignas y malignas, sin embargo muchos de los criterios utilizados son subjetivos.
La subjetividad trae como consecuencia variabilidad en la interpretación de los criterios morfológicos. La discordancia entre los microscopistas se ha tratado de subsanar estandarizando las características morfológicas y citoarquitectónicas sin embargo continúa existiendo un grado considerable de discordancia interobservador. Es recomendable que exista uniformidad en la interpretación histopatológica de las displasias epiteliales y carcinomas con el objetivo de disminuir el margen de error en el momento del diagnóstico con la finalidad de contribuir a disminuir la variabilidad interobservador, el principal objetivo del presente reporte es determinar la variabilidad intra e inter observador en el diagnóstico y graditaje de displasias epiteliales y carcinoma oral de células escamosas (COCE)

\footnotetext{
* Residente de Maestría en Ciencias Médicas, Odontológicas y de la Salud. Laboratorio de Patología Bucal Clínica y Experimental DEPeI. Universidad Nacional Autónoma de México.

** Jefe de Laboratorio de Patología Bucal Clínica y Experimental. Universidad Nacional Autónoma de México.

**** Coordinadora del Programa de Maestría y Doctorado en Ciencias Medicas, Odontológicas y de la Salud. Universidad Nacional Autónoma de México.
} 
y determinar que parámetros citológicos presentan mayor dificultad de interpretación.

\section{MATERIAL Y MÉTODO}

Se revisó el archivo del servicio de diagnóstico de patología bucal de la División de Estudios de Posgrado de la Facultad de Odontología de la UNAM, durante el periodo comprendido de enero de 1989 a diciembre de 2009 para identificar todos los casos con diagnóstico de displasia epitelial y/o carcinoma de células escamosas oral. Los casos deberían contar con suficiente material biológico para ser analizados y fueron codificados de tal forma que los observadores no conocieran el diagnóstico original o algún dato demográfico.

Un patólogo experimentado seleccionó y estandarizó los criterios morfológicos para el diagnóstico de displasias y carcinomas. Fueron revisados todos los casos al microscopio de luz por tres expertos en patología oral de manera independiente utilizando para tal fin los criterios de malignidad propuestos por la Organización Mundial de la Salud en el año 2005 y adicionalmente a los participantes se les solicitó que describieran el frente de invasión tumoral y la clasificación de Broders y que clasificaran las displasias epiteliales en leves, moderadas y severas y los carcinomas en bien, moderada y pobremente diferenciados. En las Tablas I a la III se presentan los criterios utilizados para llevar a cabo el estudio.

Se creó una base de datos ex-profeso y la comparación de datos se llevo a cabo mediante la prueba estadística Kappa interpretando los resultados bajo los criterios de Landis y Koch de cada parámetro citológico y citoarquitectónico. Utilizando para tal fin el software SPSS versión 16.

\section{RESULTADOS}

Se revisaron 18,971 archivos de los cuales se seleccionaron 220 casos con diagnóstico de displasia y carcinomas de células escamosas; la muestra estuvo conformada por 30 displasias y 30 carcinomas cálculo que se realizó utilizando el programa Sample Size Calculator. En la Tabla V mostramos los resultados obtenidos por los tres patólogos en la examinación de las displasias y en la Tabla VI el graditaje de los carcinomas del estudio de acuerdo a la clasificación de Broders.

En la valoración del frente de invasión tumoral para carcinomas se obtuvo lo siguiente:

Observador 1. Mitosis leve 16(53\%), moderada 9(30\%), severa $4(13 \%)$, nula 1(3\%)patrón de invasión leve $15(50 \%)$, moderado 10(33\%) severo 5(16\%), profundidad tumoral de invasión leve $15(50 \%)$, moderada $10(33 \%)$ severa 5(16\%), grado de queratinización: leve 13(43\%), moderado 13(43\%) severo $4(13 \%)$, pleomorfismo nuclear: leve $17(56 \%)$, moderado $10(33 \%)$ severo $3(10 \%)$, infiltrado inflamatorio: leve $7(23 \%)$, moderado 22 (73\%), y severo $1(3 \%)$.

Observador 2 . Mitosis nula 5(16\%) leve 15(50\%), moderada $10(33 \%)$, patrón de invasión leve $16(53 \%)$, moderado $8(26 \%)$, severo $6(20 \%)$, profundidad tumoral de invasión leve $15(50 \%)$, moderada $8(26 \%)$ severa $5(16 \%)$, nula $2(3 \%)$, grado de queratinización: leve $15(50 \%)$, moderado $10(33 \%)$ severo 5(16\%), pleomorfismo nuclear: leve 14(46\%), moderado $12(40 \%)$ severo $4(13 \%)$, infiltrado inflamatorio: leve $10(33 \%)$, moderado $19(63 \%)$ y severo $1(3 \%)$

Observador 3. Mitosis leve 16(53\%), moderada 10(33\%), severa $1(3.33 \%)$, nula $3(10 \%)$ patrón de invasión leve $13(43 \%)$, moderado $11(37 \%)$ severo $6(20 \%)$, profundidad tumoral de invasión leve 15(50\%), moderada 8(26\%) seve-

Tabla I. Criterios de Malignidad de la OMS.

\begin{tabular}{ll}
\hline \multicolumn{1}{c}{ Datos arquitecturales } & \multicolumn{1}{c}{ Datos Citológicos } \\
\hline E stratificación irregular & 1. Variación anormal en el tamaño nuclear \\
P érdida de la polaridad de las células basales & 2. V ariación anormal en la forma nucle ar \\
I restas epiteliales anómalas & 3. V ariación anormal en el tamaño celular \\
A umento en el numero de mitosis & 4. V ariación anormal en la forma celular \\
M itosis superficiales & 5. A umento en la proporción núcleo citoplasma \\
V ueratinización prematura de células aisladas & 6. A umento del tamaño nuclear \\
P erlas de queratina dentro de las crestas & 7. M itosis atípicas \\
& 8. A umento del número/tamaño de nucléolos \\
\hline
\end{tabular}


ra $5(16 \%)$, nula $2(6 \%)$, grado de queratinización: leve $13(43 \%)$, moderado $11(36 \%)$ severo 6(20\%), pleomorfismo nuclear: leve $15(50 \%)$, moderado $12(40 \%)$ severo $3(10 \%)$, infiltrado inflamatorio: leve 10(33\%), moderado 18 (60\%) y severo $2(6 \%)$.

El valor Kappa de concordancia intraobservador para el primer observador fue 0,90 , para el segundo de $0,91 \mathrm{y}$ para el tercero 0,92. En el caso de las displasias y los carcinomas los valores Kappa presentados se obtuvieron según la observación y diagnóstico por cada patólogo en relación con el diagnóstico estándar de oro.

\section{DISCUSIÓN}

Distintos estudios, que van desde el año 1985 hasta el 2007 (Pindborg, 1981; Abbey et al., 1995; Kujan et al., 2007; Brothwell et al.; 2003; Fisher et al., 2004) mostraron una gran variabilidad inter e intra observador en el diagnostico y gradificación de las displasias orales utilizando diferentes métodos estadísticos. El diagnóstico y el grado de displasia epitelial es un dato subjetivo y está sujeto a la valoración personal de cada observador, por ello son frecuentes las discrepancias entre los grados de displasia epitelial; la importancia de determinar la existencia de displasia epitelial en le- siones bucales, no sólo radica en que se trata de un indicador fundamental para determinar potencial de malignización, sino también para establecer la conducta a seguir.

En nuestro caso al hacer el diagnóstico y graditaje de las displasias los 3 examinadores obtuvieron una fuerza de concordancia inter observador aceptable.

En el caso de los carcinomas la concordancia inter observador fue muy buena lo que confirma que para el patólogo bucal el graditaje de las displasias representa un mayor grado de dificultad y un reto diagnóstico mayor con respecto a los carcinomas.

Sin embargo con los resultados obtenidos reconocemos una concordancia inter observador excelente debido a que los patólogos bucales recibieron una buena estandarización previa y utilizaron en el caso de los carcinomas la valoración de la clasificación de Broders y el frente de invasión tumoral lo que los obligaba a examinar los casos a detalle.

Por lo que coincidimos con Fisher et al. que destaca que la concordancia está directamente relacionada con la valoración de diversas variables una de ellas es el infiltrado inflamatorio. En nuestro caso esta variable fue tomada en cuenta en el frente de invasión tumoral.

Tabla II. Clasificación de Broders

\begin{tabular}{ll}
\hline Clasificación & de Broders \\
\hline Grado I & $25 \%$ de las células malignas están indiferenciadas \\
Grado II & $50 \%$ de las células malignas están indiferenciadas \\
Grado III & $75 \%$ o más de las células malignas están indiferenciadas \\
\hline
\end{tabular}

Tabla III. Frente de invasión tumoral (FIT).

\begin{tabular}{|c|c|c|c|c|}
\hline Número de mitosis & Nulo $=0$ & Leve de $0-1$ & Moderado de 2-5 & Severo más de 5 \\
\hline Patrón de invasión & & Cercano al vaso & Rodea vasos y nervios & Infiltra vasos y nervios \\
\hline Profundidad tumoral de invasión & & Lámina propia & Músculo y glándula & Invade hueso \\
\hline Grado de queratinización & & Más del 75\% & Hasta un $50 \%$ & Menos del $25 \%$ \\
\hline Pleomorfismo nuclear & & Menos del $25 \%$ & Del 50 al $75 \%$ & Mas del $75 \%$ \\
\hline Infiltrado inflamatorio & & Intenso & Moderado & Leve \\
\hline
\end{tabular}

\begin{tabular}{ll}
\hline$<0$ & No hay acuerdo \\
0 a 0.20 & Mínima \\
0,21 a 0,40 & Regular \\
0,41 a 0,60 & Aceptable \\
0,61 a 0,80 & Muy buena \\
0,81 a 1,00 & Excelente \\
\hline
\end{tabular}

Tabla IV. Valor de Kappa y

fuerza de concordancia. 
CUEVAS G. J. C.; MAYA G. I.; VILLANUEVA S. F. G.; GAITÁN, C. L. A. \& LEYVA, H. E. R. Estandarización en la observación y clasificación de lesiones epiteliales premalignas y malignas. Int. J. Morphol., 29(3):706-710, 2011.

Tabla V. Distribución de frecuencias para las displasias por parte de cada examinador

\section{Datos arquitecturales}

\section{Datos citológicos}

Estratificación irregular

A $20(66 \%)$

B 23(76\%)

C $22(73 \%)$

Pérdida de la polaridad de las células basales

A $23(76 \%)$

B 20(66\%)

C $25(83 \%)$

Crestas epiteliales anómalas

A $15(50 \%)$

B $18(60 \%)$

C $18(60 \%)$

Aumento en el numero de mitosis
A $13(43 \%)$
B $10(33 \%)$
C $16(53 \%)$

Mitosis superficiales

A $10(33 \%)$

B 11(36\%)

C $8(26 \%)$

Queratinización prematura de células aisladas

A $18(60 \%)$

B $18(60 \%)$

C $19(63 \%)$

Perlas de queratina dentro de las crestas
A $5(16 \%)$
B 5(16\%)
C $8(26 \%)$

Variación anormal en el tamaño nuclear
A $18(60 \%)$
B 20(66\%)
C $19(63 \%)$

Variación anormal en la forma nuclear
A $20(66 \%)$
B $15(50 \%)$
C $18(60 \%)$

Variación anormal en el tamaño celular
A $12(40 \%)$
B $14(46 \%)$
C $13(44 \%)$

Variación anormal en la forma celular
A $17(56 \%)$
B $16(53 \%)$
C $19(63 \%)$

Aumento en la proporción núcleo citoplasma

A $16(53 \%)$

B 19(63\%)

C $23(76 \%)$

Aumento del tamaño nuclear

A $14(46 \%)$

B 12(40\%)

Mitosis atípicas
A $9(30 \%)$
B $8(26 \%)$
C $8(26 \%)$

Aumento del número/tamaño de nucléolos
A $13(43 \%)$
B $9(30 \%)$
C $9(30 \%)$

Hipercromasia nuclear
A $16(53 \%)$
B 20(66\%)
C $22(73 \%)$ 
Tabla VI. Graditaje de los carcinomas por examinador.

\begin{tabular}{lccc}
\hline & Observador 1 & Observador 2 & Observador 3 \\
\hline Bien & 17 & 14 & 15 \\
Moderadamente & 10 & 12 & 12 \\
Pobremente Diferenciado & 3 & 4 & 3 \\
\hline
\end{tabular}

Tabla VII. Valores de Kappa por examinador para displasias y carcinomas.

\begin{tabular}{lccc}
\hline Observador 1 (Valor de Kappa) & Observador 2 & Observador 3 \\
\hline Carcinomas & 0,75 & 0,75 & 0,77 \\
Displasias & 0,60 & 0,59 & 0,59 \\
Intraobservad or & 0,90 & 0,91 & 0.92 \\
\hline
\end{tabular}

En el caso de la valoración intra observador los tres patólogos bucales obtuvieron una fuerza de concordancia excelente (Tabla VII) esto hace más sólidos los resultados obtenidos inter observador para cada uno de ellos de otra manera restaría importancia lo reportado inter observador si el patólogo al ver una laminilla da un diagnóstico diferente cada vez que la valora
Los patólogos participantes revisaron de manera sistemática las laminillas lo que permite no pasar por alto detalles importantes sobre todo en las displasias que son consideradas lesiones potencialmente cancerizábles.

Una de las principales razones por la que por décadas se ha reportado variabilidad en el diagnóstico de estas entidades es por la subjetividad de los parámetros a valorar sin embargo en la actualidad en patología oral y maxilofacial la biopsia y el análisis histopatológico siguen siendo el estándar de oro de las pruebas diagnósticas y un resultado confiable y preciso permitirá al clínico emplear

la mejor terapéutica para el paciente en base al diagnóstico histopatológico.

AGRADECIMIENTOS: Programa de Maestría y Doctorado en Ciencias Médicas Odontológicas y de la Salud. Universidad Nacional Autónoma de México.

CUEVAS G. J. C.; MAYA G. I.; VILlanUEVA S. F. G.; GAITÁN, C. L. A. \& LEYVA, H. E. R. Standardization in the observation and classification of premalignant and malignant epithelial lesions. Int. J. Morphol., 29(3):706-710, 2011.

SUMMARY: In the histological criteria for determining the degree of dysplasia, the Broders classification and the front of tumor invasion (FTI) are unquantifiable subjective parameters that may indicate the degree of development of carcinomas. An important factor to consider during the histological evaluation is the variability in the diagnosis of pathologists. The objective to standardize criteria and determine the intra and inter-observer variability in the diagnosis of dysplasias and OSCC. We selected and standardized morphological criteria for the diagnosis, and the cases were reviewed randomly by three oral pathologists (30 dysplasias and 30 carcinomas) from the Laboratory of Clinical and Experimental Pathology of the FO DEPeI, UNAM. Each pathologist analyzed and recorded the parameters for dysplasia and OSCC FIT on two occasions. Kappa test was applied to assess intra and inter-observer agreement. Observer $1 \mathrm{v} / \mathrm{s} 2$ match for OSCC was $0.75,0.60$ for dysplasias and intra observer 0.90 . Observer $2 \mathrm{v} / \mathrm{s} 3$ presented a concordance of 0.75 for OSCC, 0.59 for dysplasias and intra-observer 0.91 . Observer $3 \mathrm{v} / \mathrm{s}$ observer 1 for OSCC was $0.77,0.59$ for dysplasias and intraobserver 0.92. Intra observer and inter-observer concordance in OSCC were good or excellent, but in dysplasia was acceptable, confirming that its assessment showed the greatest difficulty with proper standardization we can obtain a better consensus between pathologists.

KEY WORDS: Standardized criteria; Inter-observer variability; Diagnostics variability; Epithelial lesions.

\section{REFERENCIAS BIBLIOGRÁFICAS}

Abbey, L. M.; Kaugars, G. E.; Gunsolley, J. C.; Burns, J. C.; Page, D. G.; Svirsky, J. A.; Eisenberg, E.; Krutchkoff, D. J. \& Cushing, M.. Intraexaminer And interexaminer reliability in the diagnosis of oral epithelial dysplasia. Oral Surg. Oral Med. Oral Pathol. Oral Radiol. End., 80(2):188-91, 1995.

Brothwell, D. J.; Lewis, D. W.; Bradley, G.; Leong, I.; Jordan, R. C.; Mock, D. \& Leake, J. L. Observer agreement in the grading of oral epithelial dysplasia. Community Dent. Oral Epidemiol., 31:300-5, 2003.

Fischer, D. J.; Epstein, J. B.; Morton, T. H. \& Schwartz, S. M. Interobserver reliability in the histopathologic diagnosis of oral pre-malignant and malignant lesions. J. Oral Pathologic. Med., 33(2):65-70, 2004.
Kujan, O.; Khattab, A.; Oliver, R. J.; Roberts, S. A.; Thakker, N. \& Sloan, P. Why oral histopathology suffers inter- observer variability on grading oral epithelial dysplasia: an attempt to understand the sources of variation. Oral Oncol., 43(3):224-31, 2007.

Pindborg, J. J. Cáncer y precáncer bucal. Buenos Aires, Ed. Médica Panamericana S. A., 1981.

Dirección para correspondencia:

Dr. Juan Carlos Cuevas González

Maestría en Ciencias Médicas, Odontológicas y de la Salud Laboratorio de Patología Bucal Clínica y Experimental Universidad Nacional Autónoma de México MEXICO

Email:cuevas-gonzalez@yahoo.com.mx

Recibido : 11-01-2011

Aceptado: 25-03-2011 